\title{
Effect of dimethyl sulphoxide on the crystal structure of porcine pepsin
}

\author{
Muppuru Muni Kesavulu, Sundaramoorthy Ramasubramanian, Kaza Suguna * \\ Molecular Biophysics Unit, Indian Institute of Science, Bangalore 560 012, Karnataka, India
}

\begin{abstract}
The structure of porcine pepsin crystallized in the presence of dimethyl sulphoxide has been analysed by X-ray crystallography to obtain insights into the structural events that occur at the onset of chemical denaturation of proteins. The results show that one dimethyl sulphoxide molecule occupies a site on the surface of pepsin interacting with two of its residues. An increase in the average temperature factor of pepsin in the presence of dimethyl sulphoxide has been observed indicating protein destabilization induced by the denaturant. Significant increase in the temperature factor and weakening of the electron density have been observed for the catalytic water molecule located between the active aspartates. The conformation of pepsin remains unchanged in the crystal structure. However, the enzyme assay and circular dichroism studies indicate that dimethyl sulphoxide causes a slight change in the secondary structure and complete loss of activity of pepsin in solution.
\end{abstract}

Keywords: Pepsin; Dimethyl sulphoxide; Protein denaturation

Protein denaturation is a classical phenomenon with implications in various biophysical processes. Denaturing proteins by chemicals, such as urea, guanidinium chloride, and dimethyl sulphoxide (DMSO), is one of the useful techniques for understanding different aspects of protein folding. It has been speculated that these chemicals unfold proteins by interfering with the interactions that stabilize the folded forms of proteins. However, the exact mechanism by which chemical denaturants unfold proteins is unclear.

Crystallization of substantially denatured proteins is almost impossible as they lose their compact folded structures in the denatured state. Nevertheless, it has been

\footnotetext{
is Abbreviations: DMSO, dimethyl sulphoxide; HEWL, hen egg-white lysozyme; nativepep, native pepsin; dmsopep, pepsin co-crystallized with DMSO; DHFR, dihydrofolate reductase; RNase A, ribonuclease A; RNase S, ribonuclease S; CD, circular dichroism.

* Corresponding author. Fax: +91 8023600535.

E-mail address: suguna@mbu.iisc.ernet.in (K. Suguna).
}

shown that crystallography can provide insights into the events that take place in the initial stages of the unfolding process. Structure analysis of a few proteins as complexes of denaturants has made it possible to visualize protein-denaturant interactions and the effect of denaturants on the protein structures at the onset of denaturation. In the earliest investigations of this nature $[1,2]$ it was established that urea brings about significant changes in the crystal structure of hen egg-white lysozyme (HEWL) by examining the X-ray diffraction patterns recorded for HEWL in the native form and in the presence of urea. Subsequent studies [3,4] include the effect of guanidinium chloride and DMSO as well on the structure of HEWL. Structural analyses were also carried out on $\alpha$ chymotrypsin with guanidinium chloride and urea [5], ribonuclease A (RNase A) with guanidinium chloride, dihydrofolate reductase (DHFR) in the presence of urea [6], and ribonuclease $\mathrm{S}$ (RNase $\mathrm{S}$ ) with urea [7].

In all these analyses, it has been observed that the chemical denaturants are involved in specific 
interactions with protein atoms but have little effect on the overall structures of the proteins. A few changes that occurred in some cases viz., HEWL $[3,4]$ and RNase S [7] are confined to very small local regions of the proteins. The presence of denaturants either increased or decreased (i) the stability of proteins as indicated by the change in their temperature factors and (ii) the number of ordered solvent molecules in the crystal. From the limited number of reports it is difficult to draw any general conclusions about the exact nature of the initial unfolding process. More studies of this type are required to enhance the current knowledge on the role of chemical denaturants in protein unfolding. With this view, we have selected porcine pepsin as a model protein for studying the influence of DMSO in the crystalline state. In this report, we present the structure of porcine pepsin crystallized in the presence of DMSO and its comparison with the native structure.

\section{Materials and methods}

\section{Crystallization}

Porcine pepsin obtained commercially from Sigma was used for crystallization by batch method as reported earlier [8]. For the crystallization of native pepsin, $280 \mathrm{mg} / \mathrm{ml}$ of porcine pepsin was prepared in double distilled water and the $\mathrm{pH}$ was adjusted to 3.6 with $0.5 \mathrm{M}$ sulphuric acid. The slight turbidity that appeared during the crystallization disappeared after the solution was warmed to $40{ }^{\circ} \mathrm{C}$. For crystallizing pepsin with DMSO, initially we tried soaking the native crystals in DMSO solution at various concentrations. Crystals either dissolved or did not diffract at concentrations higher than $1 \%(\mathrm{v} / \mathrm{v})$ of DMSO. Finally, we were able to obtain diffraction data with crystals soaked in 1\% DMSO solution. Co-crystallization was also performed in the presence of $1 \%$ DMSO as the protein precipitated rapidly at higher concentrations of DMSO.

\section{Data collection and processing}

Diffraction data were collected at $293 \mathrm{~K}$ using a MAR 345 imaging plate coupled to a Rigaku X-ray generator with a copper rotating anode (wavelength $=1.5418 \AA$ ) operating at $48 \mathrm{kV}$ and $100 \mathrm{~mA}$. The crystal to film distance was maintained at $300 \mathrm{~mm}$ throughout the data collection. One hundred frames, each with a 5 min exposure time, were collected with $1.0^{\circ}$ oscillation range. Diffraction data were processed and scaled using the programs DENZO and SCALEPACK [9] (see Table 1).

\section{Refinement}

As the space group and the cell parameters either in the presence or in the absence of DMSO are found to be similar to those of the reported structure [8], the refinement was initiated with the coordinates of this structure (PDB code: 5PEP, $\mathrm{P}_{5} 22, a=b=67.4$, $c=290.1 \AA$ A). Refinement was carried out using CNS program [10]. Ten percent of the total data were set aside for the calculation of $R_{\text {free }}$. Model building and electron density map examination was carried out using the program $\mathrm{O}$ [11]. Water molecules which were omitted from the initial model were added during the course of the refinement (see Table 2).
Table 1

Data collection statistics

\begin{tabular}{lll}
\hline & Dmsopep & Nativepep \\
\hline Space group & $\mathrm{P}_{5} 22$ & $\mathrm{P} 6_{5} 22$ \\
$a=b(\AA)$ & 67.47 & 67.46 \\
$c(\AA)$ & 290.29 & 290.59 \\
Maximum resolution $(\AA)$ & 3.0 & 3.0 \\
Overall $R_{\text {merge }}(\%)$ & 7.8 & 63 \\
$R_{\text {merge last shell }(3.11-3.0 \AA)(\%)}$ & 17.6 & 120 \\
Overall completeness $(\%)$ & 93.7 & 99.2 \\
Completeness in the last shell & 91.1 & 98.6 \\
$\quad(3.11-3.0 \AA)(\%)$ & & \\
\hline
\end{tabular}

Table 2

Refinement statistics

\begin{tabular}{lll}
\hline & Dmsopep & Nativepep \\
\hline Total number of reflections & 7706 & 8405 \\
Number of reflections for evaluation of $R_{\text {free }}$ & 829 & 896 \\
Resolution $(\AA)$ & $20.0-3.0$ & $20-3.0$ \\
Number of atoms & & \\
$\quad$ Protein & 2429 & 2426 \\
$\quad$ Water & 189 & 90 \\
$\quad$ Denaturant & 4 & - \\
Final $R$-factor $(\%)$ & 18.7 & 18.4 \\
Final $R_{\text {free }}(\%)$ & 28.0 & 25.05 \\
Average $B\left(\AA^{2}\right)$ for all atoms & 44.60 & 29.04 \\
\hline
\end{tabular}

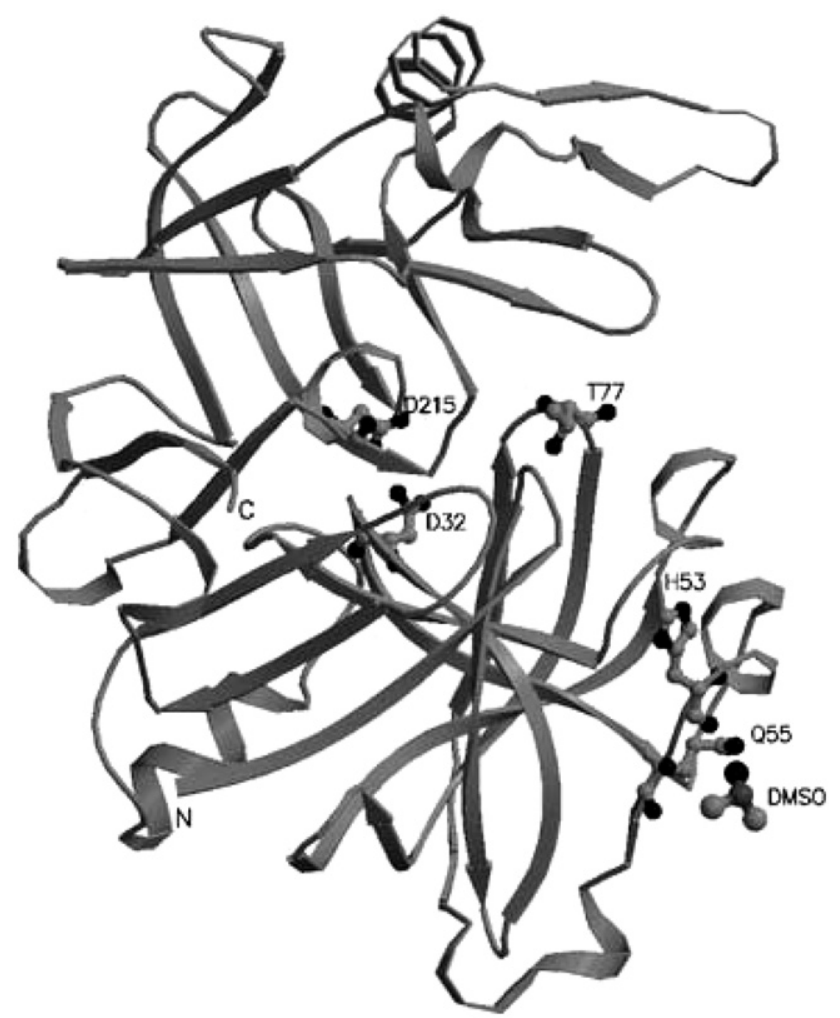

Fig. 1. The backbone trace of porcine pepsin showing the active aspartates, D32 and D215; a residue T77 at the tip of the flexible 'flap' region; and the DMSO molecule and the two residues H53 and Q55 that interact with DMSO. This figure and Figs. 2 and 3 have been generated using the program MOLSCRIPT [15] and rendered using RASTER3D [16]. 


\section{Solution studies}

Circular dichroism spectroscopy. Circular dichroism (CD) spectra were recorded on the JASCO J-715 spectropolarimeter in aqueous solution containing $0.28 \mathrm{mg} / \mathrm{ml}$ of porcine pepsin $(8 \mu \mathrm{M})$ and for the same solution containing $1 \%$ DMSO. The wavelength range used was $195-250 \mathrm{~nm}$ at RT using a $0.1 \mathrm{~cm}$ path length CD cuvette. The spectra were corrected by subtracting the corresponding solvent blank.

Pepsin assay. To check the activity of pepsin in the presence of DMSO in solution, pepsin assay was performed in the presence and absence of DMSO. Equine haemoglobin was used as the substrate, and the assay was performed by altering the method described in the commercial web site [12] based on the method of Anson [13].

\section{Results}

Both native pepsin (nativepep) and pepsin co-crystallized with DMSO (dmsopep) crystals appeared within 2 days and reached the maximum size within 7 days. The crystals of dmsopep tend to grow to sizes much bigger than those of nativepep. However, the biggest dmsopep crystals $(\sim 1 \mathrm{~mm}$ in length) were twinned as apparent from the diffraction data. Data were collected using

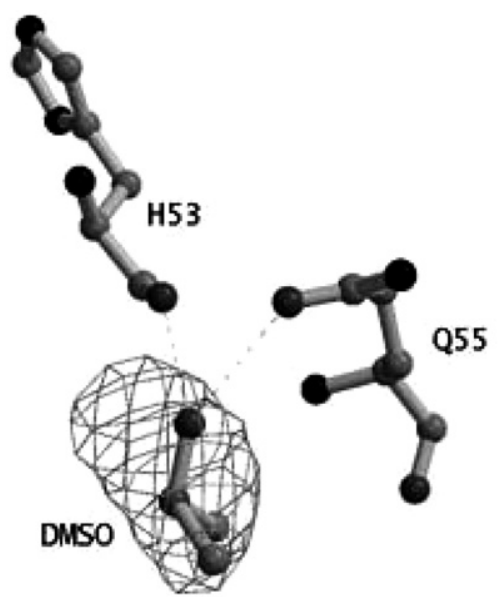

Fig. 2. Electron density of the $2 f_{\mathrm{o}}-f_{\mathrm{c}}$ map contoured at $3 \sigma$ level for the DMSO molecule. The interaction between DMSO and the two residues of pepsin H53 and Q55 is indicated as dashed lines. nativepep crystals of the size $0.36 \times 0.20 \times 0.06 \mathrm{~mm}$ and a dmsopep crystal of the size $0.52 \times 0.22 \times 0.06 \mathrm{~mm}$.

The available structure of porcine pepsin was determined using synchrotron data to a resolution of $2.34 \AA$ [8]. The crystals we grew diffracted to $3.0 \AA$ resolution at home source. Diffraction data were collected and refinement was carried out for the native pepsin also such that the structures processed in the same conditions can be compared for the analysis.

We could not locate DMSO or observe any other changes in the structure on further analysis using the data collected on the crystals soaked in DMSO solution. The structure of dmsopep that is discussed here is that of pepsin co-crystallized with DMSO.

All the 316 residues as in 5PEP could be clearly seen in the electron density maps of nativepep and dmsopep. Assignment of the 'ordered' water molecules was done if clear density appeared above $1 \sigma$ level in the $2 f_{\mathrm{o}}-f_{\mathrm{c}}$ maps and above $3 \sigma$ level in the $f_{\mathrm{o}}-f_{\mathrm{c}}$ maps. Of the 371 water molecules present in 5PEP, we could locate only 89 in the nativepep structure, whereas 170 common waters were located in dmsopep. Only 60 waters are common between the structures of nativepep and dmsopep. In addition to 170 waters in dmsopep, we found 19 new waters, which were not present in the native pepsin crystal structures. One water was found both in nativepep and dmsopep which was not present in 5PEP. Towards the final stages of the refinement with the inclusion of most of the water molecules, strong positive density was observed in the $f_{\mathrm{o}}-f_{\mathrm{c}}$ difference electron density map of dmsopep above $5 \sigma$ cutoff value. The shape of the density also appeared to be different from that for a water molecule. A DMSO molecule was placed at this position and refined. The coordinates and the structure factors of dmsopep have been deposited in the Protein Data Bank (PDB code: Iyx9).

DMSO occupies a site on the surface of the protein close to His53 and Gln55 as shown in Figs. 1 and 2. The oxygen atom of DMSO is within interacting distance with the carbonyl oxygen of His $53(3.23 \AA)$ and OE1 atom of Gln55 (2.88 $⿱$ ). In addition, DMSO makes four van der Waals contacts with this region (His53,
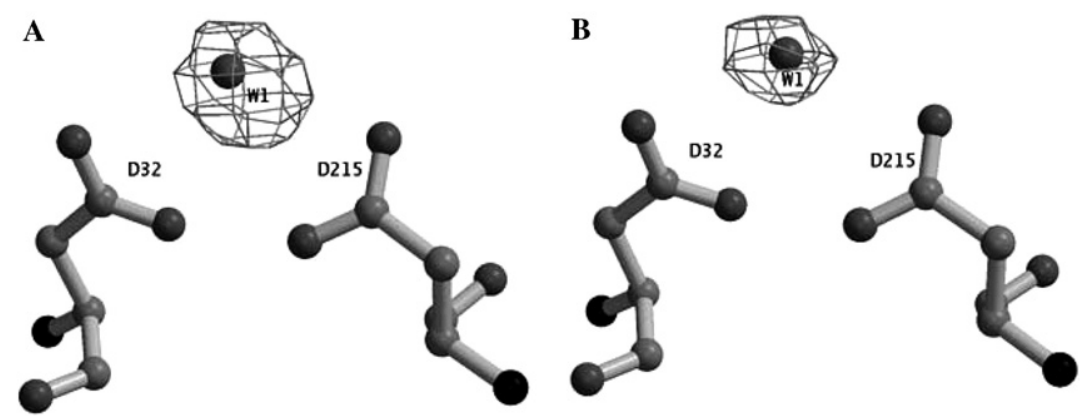

Fig. 3. Electron density of the $2 f_{\mathrm{o}}-f_{\mathrm{c}}$ map at the catalytic water molecule contoured at $3 \sigma$ level in (A) native pepsin and (B) pepsin + DMSO complex. 


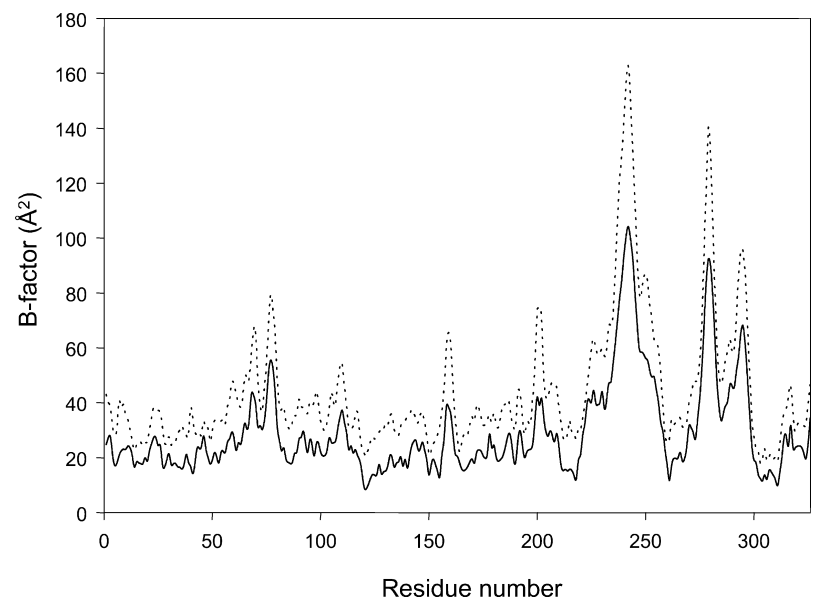

Fig. 4. A plot of the $B$-factors of the $\mathrm{C}^{\alpha}$ atoms of porcine pepsin. The solid line represents native pepsin and the dotted line represents pepsin + DMSO complex.

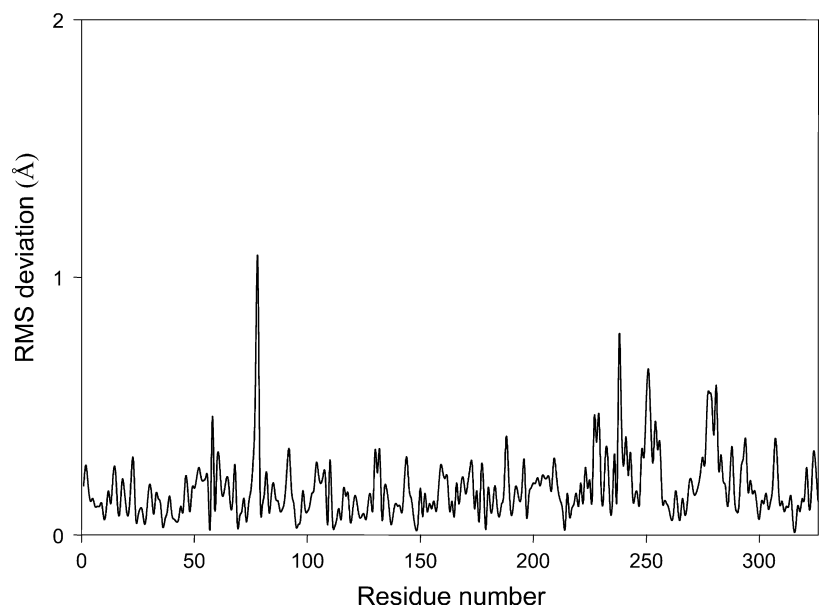

Fig. 5. RMS deviation of the positions $\mathrm{C}^{\alpha}$ atoms of the pepsin + DMSO complex from those in the native pepsin structure.

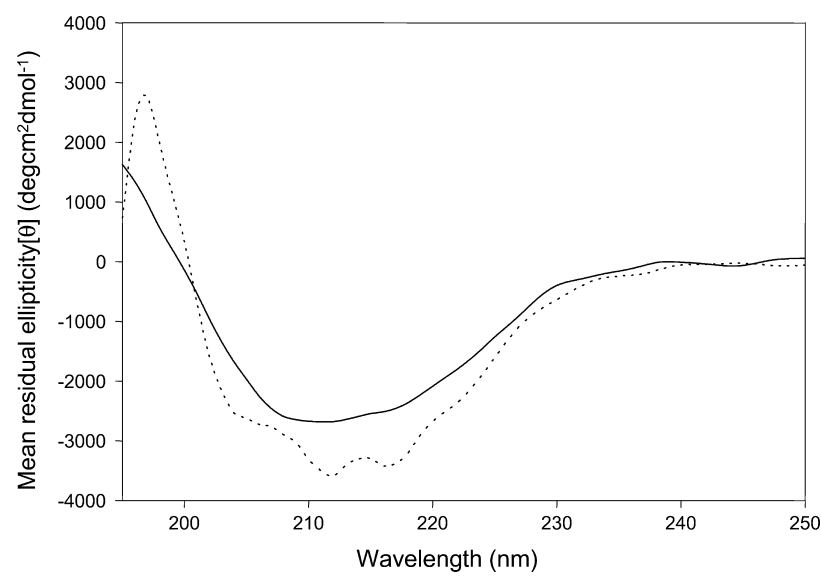

Fig. 6. The CD spectra of the native pepsin (solid line) and pepsin + DMSO complex (dotted line).
Asn54, and Gln55) and two contacts with a nearby water molecule (Water 30). DMSO occupies this position displacing one water molecule present in the native structure, which does not make any interactions with the protein. The electron density for the catalytic water present between the two aspartic acid residues (Asp32 and Asp215) appears to be much weaker in the dmsopep structure compared to the density in nativepep (Figs. 3A and B). The electron density for this water appeared only towards the end of the refinement. The temperature factor of this water molecule also increases significantly in the presence of DMSO from 6.42 to $32.13 \AA^{2}$.

The temperature factors ( $B$-factors) of the $C^{\alpha}$ atoms of both nativepep and dmsopep are shown in Fig. 4. Comparison of the average $B$-factors showed significantly higher values for dmsopep structure for the protein atoms $\left(\mathrm{C}^{\alpha}\right.$ atoms 44.04; all atoms $\left.43.46 \AA^{2}\right)$ and for the common water molecules $\left(40.92 \AA^{2}\right)$ compared to those in nativepep $\left(C^{\alpha}\right.$ atoms 29.13 ; all atoms 29.04; waters $31.42 \AA^{2}$ ). When the nativepep and dmsopep structures were superimposed using the program ALIGN [14], the rmsd between these two structures for all the protein atoms was found to be $0.19 \AA$ (Fig. 5).

To study the effect of DMSO on the secondary structure of porcine pepsin, we have recorded the CD spectra (Fig. 6). The spectra show that the addition of DMSO to porcine pepsin results in a modest increase in the secondary structural component. The enzyme assay experiments using haemoglobin showed complete loss of pepsin activity by the addition of $1 \%$ DMSO.

\section{Discussion}

In the present study, the crystal structure of porcine pepsin has been determined in the presence of DMSO in an attempt to characterize the protein-denaturant interactions and to find the effect of chemical denaturants at the initiation of protein denaturation. As observed in the previous studies [3-7], DMSO makes specific interactions with protein atoms without affecting the overall conformation of the protein. The rmsd of $0.19 \AA$ between the nativepep and dmsopep structures is small compared to that observed for some of the reported structures but the rmsd values had a tendency to increase with higher concentration and/or number of denaturant molecules found to be bound to the protein [3]. The only region that shows a significant shift is near Thr77 (Fig. 1) of the flexible 'flap' region. This loop is known to have high mobility as it is involved in the substrate binding, in aspartic proteinases. The differences in the secondary structure between nativepep and dmsopep as found from the CD spectra do not correlate with the crystal structure studies as the structure remains the same in both the crystals. In solution, 
DMSO will most likely have more interactions with the protein which in turn will alter the protein structure.

The average temperature factor reflects the static and/or the dynamic disorder of the protein molecules in the crystal lattice. Higher flexibility or mobility or loose packing makes the proteins disordered and less stable resulting in higher average temperature factors. As the nativepep and dmsopep crystallize in the same space group, the increase in the average temperature factor of about $15 \AA^{2}$ for the protein atoms in dmsopep compared to that in nativepep cannot be due to differences in packing but due to an increase in the mobility of the protein in the presence of DMSO. Similar to this, it was observed that the average $B$-factors increased due to the presence of denaturants in RNase S with urea [7] and HEWL with DMSO [4]. In contrast, in the studies on HEWL with urea [3], RNase A with guanidinium chloride, and DHFR with urea [6], it was observed that binding of denaturants causes a decrease in the $B$-factors. Another variation that has been noted is the way in which the denaturants affect the solvent structure in proteins. Only in the structure of HEWL with urea [3], RNase A with guanidinium chloride, and DHFR with urea [6], the number of water molecules increased after the binding of denaturant. We also have observed the appearance of 19 new waters in dmsopep structure. The denaturants appear to be modifying the protein stability and the solvent structure of different proteins in different ways.

An interesting observation from this study is the weakening of the electron density and an increase in the temperature factor for the catalytic water with the addition of DMSO. The $B$-factor increased from 6.42 to $32.13 \AA^{2}$. It is rather surprising as the catalytic site is not very close to the DMSO binding site (Fig. 1). It indicates that the denaturation set by DMSO might be triggering the loss of enzyme activity. Once the protein is free from the confines of the crystal it may denature completely and become totally inactive as observed from the enzyme assay study in solution.

\section{Acknowledgments}

Intensity data were collected at the X-ray Facility for Structural Biology supported by the Department of Science and Technology (DST) and the Department of Biotechnology (DBT). Computations were carried out at the Bioinformatics Centre and Graphics Facility (both supported by DBT) and the Supercomputer Education and Research Centre at the Institute. The work is funded by the Council of Scientific and Industrial Research.

\section{References}

[1] J. Berthou, P. Jolles, The influence of urea on crystallization and polymorphism of hen lysozyme, FEBS Lett. 31 (1973) 189-192.

[2] K.W. Snape, R. Tjian, C.C.F. Blake, D.E. Koshland, Crystallographic study of the interaction of urea with lysozyme, Nature 250 (1974) 295-298.

[3] A.C.W. Pike, K.R. Acharya, A structural basis for the interaction of urea with lysozyme, Protein Sci. 3 (1994) 706-710.

[4] S.C. Mande, M.E. Sobhia, Structural characterization of proteindenaturant interactions: crystal structures of hen egg-white lysozyme in complex with DMSO and guanidinium chloride, Protein Eng. 13 (2000) 133-141.

[5] L.S. Hibbard, A. Tulinsky, Expression of functionality of $\alpha$-chymotrypsin. Effects of guanidine hydrochloride and urea in the onset of denaturation, Biochemistry 17 (1978) 5460-5468.

[6] J. Dunbar, H.P. Yennawar, S. Banerjee, J. Luo, G.K. Farber, The effect of denaturants on protein structure, Protein Sci. 6 (1997) 1727-1733.

[7] G.S. Ratnaparkhi, R. Varadarajan, X-ray crystallographic studies of the denaturation of ribonuclease S, Proteins: Struct. Funct. Genet. 36 (1999) 282-294

[8] J.B. Cooper, G. Khan, G. Taylor, I.J. Tickle, T.L. Blundell, Xray analysis of aspartic proteinases. II. Three-dimensional structure of the hexagonal crystal form of porcine pepsin at $2.3 \AA$ resolution, J. Mol. Biol. 214 (1990) 199-222.

[9] Z. Otwinowski, W. Minor, Processing of X-ray diffraction data collected in oscillation mode, Methods Enzymol. 276 (1997) 307326.

[10] A.T. Brunger, P.D. Adams, G.M. Clore, W.L. DeLano, P. Gros, R.W. Grosse-Kunstleve, J.S. Jiang, J. Kuszewski, M. Nilges, N.S. Pannu, R.J. Read, L.M. Rice, T. Simonson, G.L. Warren, Crystallography and NMR system: a new software suite for macro molecular structure determination, Acta Crystallogr. D 54 (1998) 905-921.

[11] T.A. Jones, J.Y. Zou, S.W. Cowan, M. Kjeldgaard, Improved methods for building protein models in electron density maps and the location of errors in these models, Acta Crystallogr. A 47 (1991) 110-119.

[12] Available from: <http://www.worthington-biochem.com/PM/ default.html>.

[13] M. Anson, The estimation of pepsin, trypsin, papain and cathepsin with hemoglobin, J. Gen. Physiol. 22 (1938) 79-89.

[14] G.E. Cohen, ALIGN: a program to superimpose protein coordinates, accounting for insertions and deletions, J. Appl. Crystallogr. 30 (1997) 1160-1161.

[15] R.M. Esnouf, An extensively modified version of MolScript that includes greatly enhanced coloring capabilities, J. Mol. Graph. 15 (1997) 132-134.

[16] E.A. Merritt, D.J. Bacon, Raster3D: photorealistic molecular graphics, Methods Enzymol. 277 (1997) 505-524. 